Compréhension du DDMRP et de son adoption : premiers éléments empiriques

First insights in DDMRP: how does it work and why companies choose it ?

Baptiste BAHU ${ }^{\mathrm{a}}$, Laurent BIRONNEAU ${ }^{\mathrm{a}}$ et Vincent HOVELAQUE ${ }^{\mathrm{a}}$

${ }^{a}$ Univ Rennes, CNRS, CREM - UMR 6211, F-35000 Rennes, France

Mail : baptiste.bahu@univ-rennes1.fr

Mail : laurent.bironneau@univ-rennes1.fr

Mail : vincent.hovelaque@ univ-rennes1.fr

Remerciement : Les auteurs remercient vivement Laurent Vigouroux (B2Wise France) pour son accompagnement dans la mise en contact avec les praticiens ainsi que l'apport de son expertise sur la méthode DDMRP. Les auteurs souhaitent également remercier les évaluateurs anonymes pour leurs suggestions qui ont permis l'apport de modifications contribuant à améliorer la clarté du propos de cet article. 


\section{Compréhension du DDMRP et de son adoption : premiers éléments empiriques}

Le Demand Driven Material Requirements Planning (DDMRP) est une méthode récente de gestion de production dont la presse professionnelle met régulièrement en avant les exemples d'implantations réussies en entreprise. Elle propose une alternative aux méthodes traditionnelles de gestion de la production, en se basant sur un dimensionnement dynamique de stocks positionnés à des points stratégiques de découplage et un fonctionnement tiré par la demande réelle. Le lancement des productions, des achats ou des transferts inter-sites ne se fait plus directement à partir de prévisions, mais selon un planning visuel adapté en temps réel, basé sur des codes couleurs de criticité et de priorité : vert, jaune ou rouge. L'objet de cet article est de décrire le fonctionnement de cette méthode, de définir ses limites, et de comprendre, à travers l'étude de 30 cas pratiques, les raisons poussant les entreprises à l'implémenter.

Demand Driven Material Requirements Planning (DDMRP) is a recent production planning and control (PPC) system. It is a subject of interest to the professional press. Indeed, numerous articles highlights cases where the implementation of the DDMRP has succeeded. The demand-driven system and the dynamic dimensioning of decoupled points (resulting from a strategic inventory positioning) bring the DDMRP as an alternative to the traditional PPC system. Forecasts are no longer the signal to release the production, purchase and intra company stock transfer order. Instead, DDMRP provides signals based on priority and critically colors: green, yellow and red; collected in a visual planning updated in real-time. The aim of this article is to describe how this method works, define its limits, and understand the reasons why companies choose to implement it.

Mots-clés : DDMRP, Gestion de la production, Gestion des stocks, Prévisions Keywords: DDMRP, Production control, Inventory management, Forecasting 


\section{Introduction}

L'environnement industriel se caractérise désormais dans de nombreuses organisations par plus (i) de volatilité (Volatility), les rythmes des changements s'accélérant, (ii) d'incertitude (Uncertainty), le volume et la diversité des informations accessibles rendant encore plus difficile le processus de prévision, (iii) de complexité (Complexity) en raison d'une multiplication importante des facteurs influençant les décisions et (iv) d'ambiguïté (Ambiguity) car il est parfois difficile de comprendre les événements du passé. Face à ce contexte défini par l'acronyme VUCA, concept né en environnement militaire et récemment étendu aux organisations et supply chain (Packowski, 2013 ; Bennett et Lemoine, 2014), deux praticiens, Carol Ptak et Chad Smith, proposent dans deux ouvrages $(2011,2016)$, une nouvelle méthode de gestion de production baptisée le DDMRP, pour Demand Driven Material Requirements Planning.

La presse professionnelle (en particulier française) semble s'être emparée de cette méthode. Le DDMRP y est notamment présenté comme l'une des 5 tendances principales des prochaines années en supply chain (Les Echos, 17 septembre 2018) et de nombreux articles décrivent des expériences concluantes d'entreprises utilisatrices en termes d'accélération du flux, d'amélioration du taux de service, de baisse du niveau des stocks, de maitrise du besoin en fond de roulement (BFR) et de réduction des délais de livraison. La méthode séduit également certains cabinets de conseil, mais aussi des associations professionnelles comme FAPICS (Association Française de Supply Chain Management), qui proposent, à destination des entreprises et des consultants, des formations préparant 
aux certifications proposées par le Demand Driven Institute (DDI) ${ }^{1}$. Quant au monde académique, il s'approprie aussi progressivement la thématique depuis 2015.

L'objet de cet article est de présenter le fonctionnement pratique de cette méthode et de proposer des propositions concernant les raisons qui semblent pousser les entreprises à s'y intéresser, travail qui semble porteur et original au regard de l'intérêt suscité par cette méthode. Ainsi, après avoir exposé la manière dont Carol Ptak et Chad Smith justifient le recours à cette nouvelle méthode, nous en expliquerons le fonctionnement $(i)$. Puis nous présenterons un état de l'art recensant les travaux académiques traitant du DDMRP ainsi que la démarche méthodologique empruntée (ii). Nous mettrons alors en avant les premiers résultats de notre étude basée sur l'analyse d'une trentaine de cas pratiques (iii) et nous conclurons par une discussion critique des arguments avancés par les auteurs tout en évoquant les perspectives de recherche qui en découlent (iv).

\section{Le DDMRP, une nouvelle méthode de pilotage au service d'une prise de décision opérationnelle}

Pour bâtir leur argumentaire sur le DDMRP, Ptak et Smith (2016) mettent en avant les limites des méthodes classiques (qu'ils résument au MRP et au Lean², lui-même réduit au Kanban) dans un environnement VUCA. Tout d'abord, ils clament que les méthodes

1. Organisme fondé en 2011 par Carol Ptak et Chad Smith, il représente l'autorité mondiale pour la formation, la certification et la conformité des méthodes «Demand Driven». https://www.demanddriveninstitute.com/

2. "The basic elements of this alternative design can be better understood by exploring a chronic conflict between two camps - the believers in Lean methodology and the believers in MRP" (Ptak et Smith, 2016, 45). "When it comes to the protection and promotion of the flow of relevant information and materials, both Lean and MRP have weaknesses in today's more volatile and complex environments." (Ptak et Smith, 2016, 50). 
MRP reposent sur le concept d'interdépendance des articles tout au long d'une chaîne et sur un calcul des besoins à partir d'historiques ou de prévisions. Le MRP serait ainsi générateur d'effets «coup de fouet $»^{3}$, dit effets Bull-Whip (Lee, Padmanabhan et Seungjin, 2004), créant une situation où le stock passe rapidement d'une rupture à un stock excédentaire (Wang et Disney, 2016 ; Mackelprang et Malhotra, 2015). Cet effet coup de fouet pourrait provenir (i) de l'aval (demand effect) et affecter l'amont tout comme (ii) de l'amont (supply effect) et générer des dysfonctionnements vers l'aval. Quant aux méthodes comme le Kanban (Schonberger, 1983 ; Ohno, 1989), qui se basent à contrario sur une logique d'indépendance des articles le long d'une chaîne et sur un pilotage basé sur une consommation réelle (et non plus sur une prévision), elles leur semblent également inadaptées pour synchroniser les flux dans l'environnement économique actuel, n'étant utiles, pour eux, qu'au sein de systèmes où la demande est relativement régulière et stable.

Ptak et Smith $(2011,2016)$ présentent le DDMRP comme une solution à ces limites. Ils l'introduisent comme une méthode se basant sur une combinaison des meilleures pratiques du MRP et du Lean, mais aussi d'autres approches comme le Six Sigma, la Théorie des Contraintes (Goldratt et Cox, 1993) ou le DRP (Distribution Ressource Planning), tout en y ajoutant quelques innovations. Elle s'intègre à un système décisionnel global (figure 1), dont les fondamentaux théoriques se précisent progressivement au sein du DDI. Le DDMRP fait ainsi partie du Demand Driven Operating Model (DDOM), décrit pour la première fois par Smith et Smith (2013). Ce dernier intègre en plus du DDMRP, considéré comme l'élément moteur, le Demand

3. Un changement extrême au point le plus en amont d'une supply chain va être généré par un petit changement de la demande en aval (Forrester, 1961). 
Driven Capacity Schedulling, qui réalise, en fonction des capacités, l'ordonnancement détaillé des ordres de fabrication à lancer, et le Demand Driven Execution qui gère les ordres lancés. Certaines des données d'entrées pour le paramétrage du DDOM sont produites par le Demand Driven Sales and Opérations Planning (DDS\&OP) qui est présenté par Ptak et Smith (2016) comme un outil de décision tactique permettant de réconcilier les décisions prises entre les niveaux opérationnels (DDOM) et stratégiques (Adaptive $S \& O P$ ). Cette synchronisation est décrite succinctement dans une note récente (Smith, Ptak et Ling, 2017) du Demand Driven Institute. Nous ne traiterons pas ces aspects dans cet article et nous nous focaliserons sur la méthode DDMRP.

Figure 1. Le système décisionnel Demand Driven (adapté de Smith, Ptak et Ling, 2017).

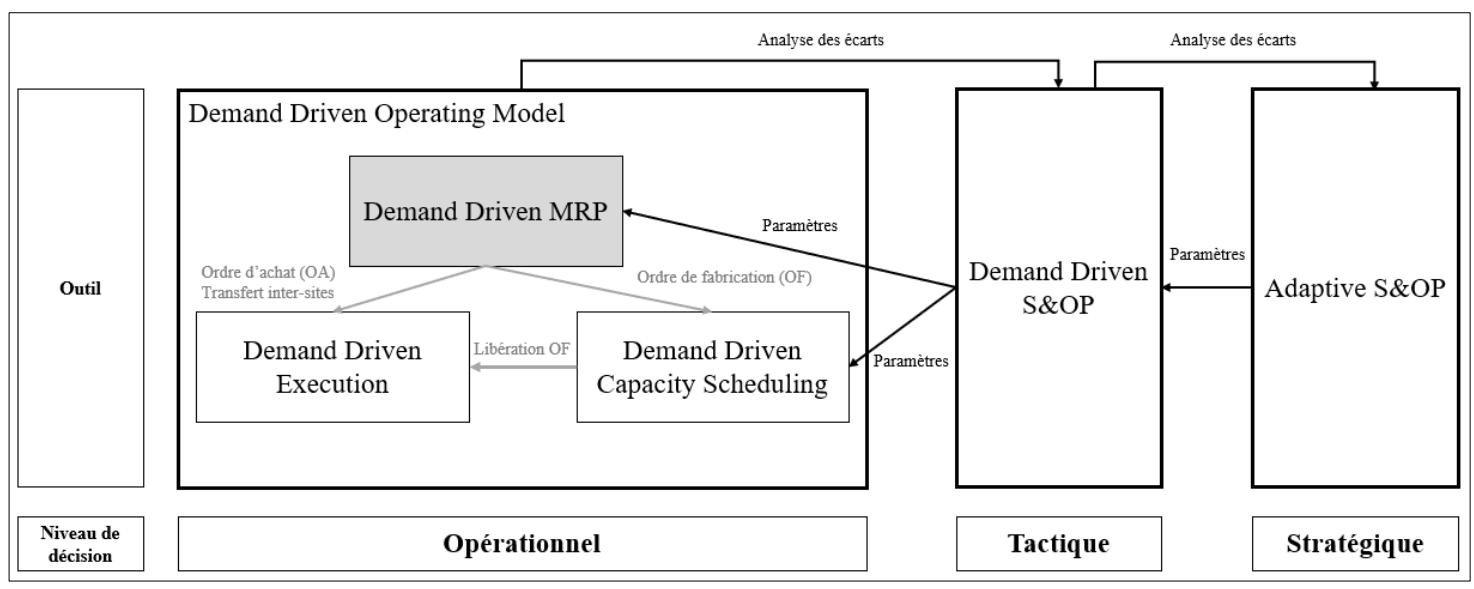

Cette méthode, dont la mise en œuvre est scindée en 5 étapes successives, (figure 2) peut être déployée au niveau de l'approvisionnement, la production et la distribution d'une organisation.

Figure 2. Les 5 cinq étapes du DDMRP (adapté de Ptak et Smith, 2016, 53). 


\begin{tabular}{|c|c|c|c|c|}
\hline \multicolumn{5}{|c|}{ Demand Driven Material Requirements Planning } \\
\hline $\begin{array}{c}\text { Positionnement } \\
\text { des stocks } \\
\text { stratégiques }\end{array}$ & $\begin{array}{c}\text { Profils de buffer } \\
\text { et niveaux }\end{array}$ & $\begin{array}{c}\text { Ajustements } \\
\text { dynamiques }\end{array}$ & $\begin{array}{c}\text { Planification } \\
\text { pilotée par la } \\
\text { demande }\end{array}$ & $\begin{array}{c}\text { Exécution } \\
\text { visible et } \\
\text { collaborative }\end{array}$ \\
\hline Positionner & \multicolumn{3}{|c|}{ Protéger } & \multicolumn{3}{|c|}{} \\
\hline
\end{tabular}

\section{Configuration initiale et évolution du modèle DDMRP - les étapes de 1 à 3 de la méthode}

Les trois premières étapes peuvent être assimilées à une phase de «paramétrage » nécessaire à l'utilisation opérationnelle du DDMRP dans les organisations.

L'étape initiale (étape 1) consiste à positionner des buffers de stocks $^{4}$ pour constituer des points de découplage permettant de rendre indépendant les différents processus. Ce découplage a un impact sur l'ordonnancement de la production d'une organisation, et potentiellement sur l'ensemble des flux logistiques d'une supply chain. En effet, la démarche peut être étendue en dehors de l'organisation afin que les fournisseurs soient connectés aux clients et déterminent leurs productions et livraisons suivant l'évolution des buffers. Il s'agit donc de déterminer les endroits, dans la chaîne logistique et dans la nomenclature d'un produit fini, qui peuvent être considérés comme étant stratégiques pour positionner ces stocks : les endroits où les stocks deviennent une source de valeur ajoutée pour l'entreprise. Les auteurs proposent une grille d'analyse

4. En plus de ce type de buffer, Smith et Smith (2013) recommandent aussi des buffers dits de temps et de capacités, n'intervenant pas directement dans le DDMRP mais dans le DDOM. Notre périmètre étant restreint à cette méthode, seuls les buffers de stocks seront traités ici. 
composée de 6 facteurs appelés « facteurs de positionnement »(Ptak et Smith, 2016, 57) pour identifier ces endroits stratégiques : (1) le délai attendu par les clients, (2) le délai d'opportunité de marché, (3) l'horizon de visibilité des commandes de ventes, (4) la variabilité externe, (5) le point de levier et de flexibilité du stock et (6) la protection des opérations critiques. Il est préconisé de réaliser une adaptation du positionnement initial des points de découplages selon un processus itératif en ajoutant de nouveaux buffers et/ou en éliminant ceux ne s'avérant pas être nécessaires. Une autre solution proposée par Rim, Jiang et Lee (2014), et Jiang et Rim (2017) consisterait à mobiliser un algorithme génétique permettant une disposition adéquate de ces points de découplage. D'après les auteurs, cette approche convient à un environnement de production à la commande au sein duquel un grand nombre de références peut exister. Pour cela l'algorithme utilise une notion apportée par la méthode DDMRP : le délai découplé (Decoupled Lead Time DLT), qui représente le délai cumulé défini comme étant le plus long délai sans buffer sur le chemin d'une nomenclature. Le positionnement des buffers est dynamique car il évolue en fonction des performances de l'ensemble de la chaine logistique.

La seconde étape du DDMRP (étape 2) consiste à dimensionner les buffers de stocks. Ils doivent pouvoir absorber différentes sources de variabilités (de la demande, des approvisionnements, du management et des opérations ${ }^{5}$ ) et garantir une rentabilité économique. Différents profils de buffer (36) sont définis selon trois paramètres (Ptak et

5. Définis par Ptak et Smith (2011) et complétés par Smith et Smith (2013), il est possible de regrouper ces 4 types de variabilité en 2 catégories: forme externe (demande et approvisionnement) et forme interne (opération et management). Nous associons ces notions de variabilité des opérations et du management à celles de « common causes » et de « special causes » identifiées par Deming (1975). La variabilité des opérations est inhérente au système, elle est aléatoire; quant à la variabilité du management, elle est due à l'intervention humaine. 
Smith, 2016, 98-105) : le type d'article (acheté, fabriqué, distribué, semi-fini), la catégorie de délai (de court à long), et la catégorie de variabilité (de faible à haute). Chaque buffer de stock est constitué de trois zones de couleur, verte, jaune et rouge, faisant chacune l'objet d'un calcul spécifique intégrant le profil de buffer et des attributs de l'article (tableau 1). La consommation moyenne journalière (CMJ), le délai découplé (DLT) et la quantité minimale à commander (MOQ : Minimum Order Quantity) constituent les attributs d'un article. Une donnée supplémentaire, correspondant au cycle de commande désiré ou imposé (nombre de jours entre deux commandes), est utilisée pour le dimensionnement de la zone verte. Cette donnée permet de prendre en compte les contraintes inhérentes aux spécificités de la politique d'approvisionnement de l'entreprise.

Tableau 1. Fonctions et formules de calcul des trois zones d'un buffer (Ptak et Smith, 2016).

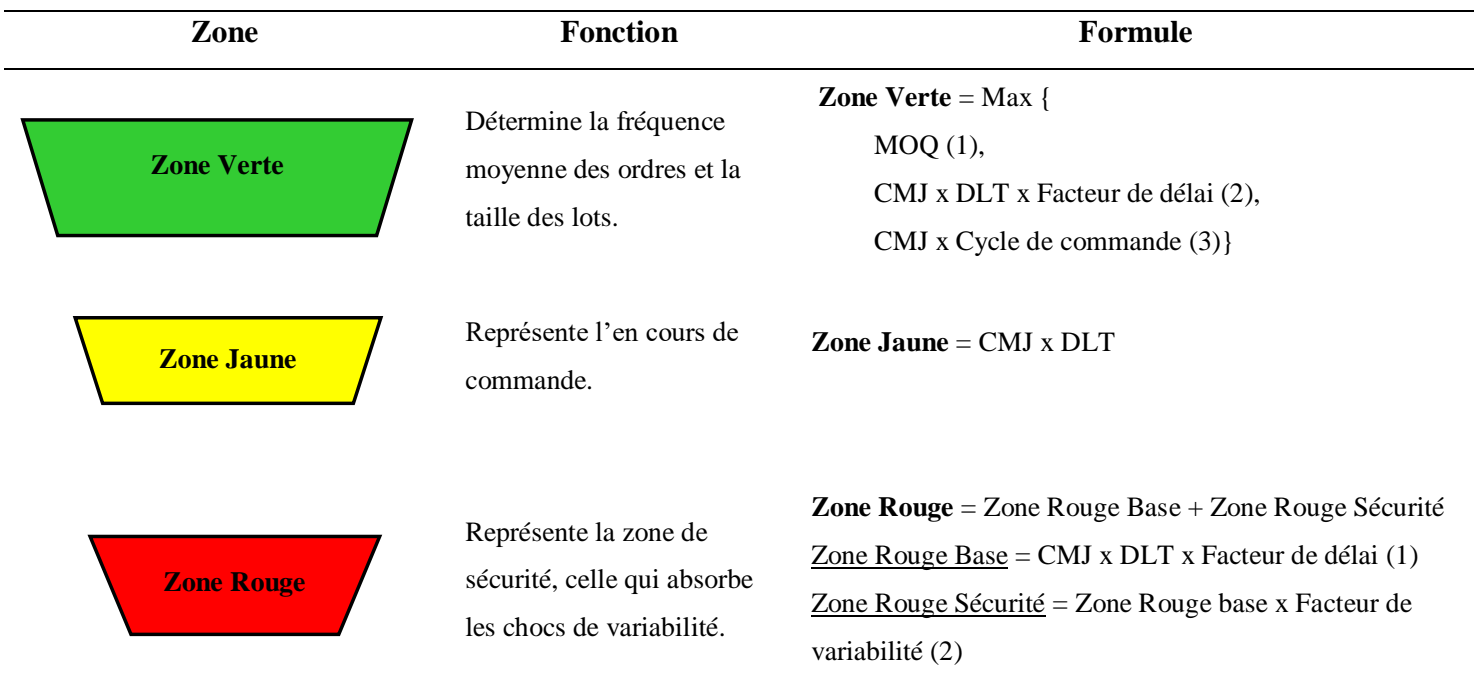


L'exemple 1 suivant permet d'expliciter le calcul :

Exemple 1. Dimensionnement détaillé d'un buffer de stock.

Soit un article A produit avec un délai et une variabilité considérée comme moyens et dont le délai de fabrication
est de 5 jours et la consommation moyenne journalière de 10 unités. Le minimum à commander est de 40 unités
pour un cycle de commande désiré de 3 jours.
Le dimensionnement du buffer est le suivant:

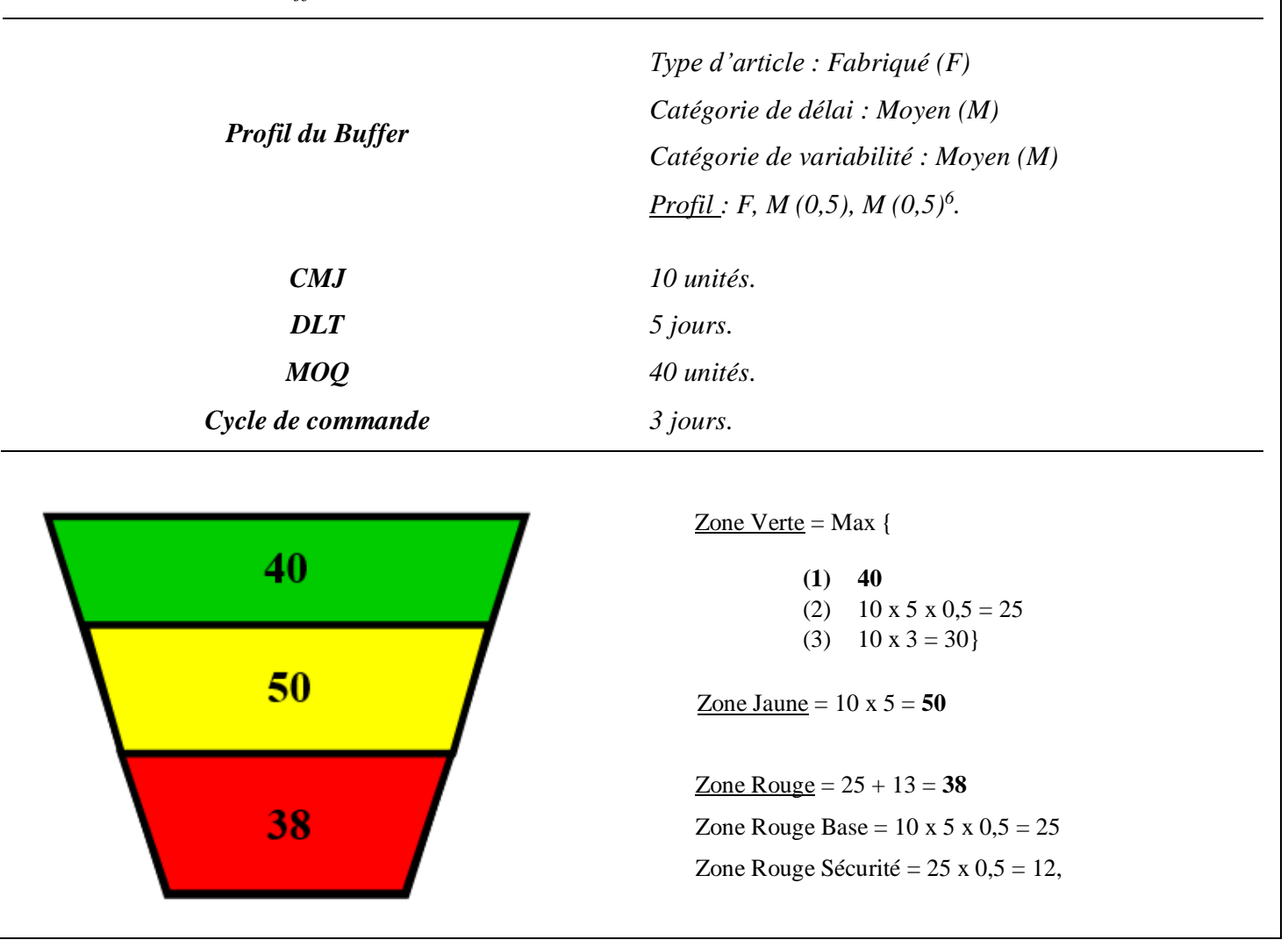

La consommation moyenne journalière, variable utilisée pour le calcul de l'ensemble des zones, est au cœur du dimensionnement des buffers de stocks. Elle est

6. Le profil du buffer permet de déterminer le facteur de délai et de variabilité. Dans cet exemple, puisque le délai est considéré comme moyen, le facteur de délai est compris entre 0,41 et 0,60 et puisque la variabilité est considérée comme moyenne, le facteur de variabilité est aussi compris entre 0,41 et 0,60 . Chaque facteur, devant être compris entre ces intervalles, est ensuite choisi empiriquement par l'entreprise. 
obtenue $^{7}$ soit par un historique de consommation défini par l'entreprise, soit par des prévisions ou encore par une combinaison de ces deux approches. Une moyenne glissante, des différentes données d'entrée, est alors réalisée pour déterminer la CMJ qui est périodiquement recalculée selon une fréquence également définie par l'entreprise. Les facteurs de délai et de variabilité sont quant à eux déterminés selon le profil de buffer de l'article. Ptak et Smith $(2011,415-416)$ propose un intervalle de valeurs pour ces facteurs, mais sans donner d'indication quant à la façon de déterminer la valeur qui mènerait vers une solution optimale. Miclo (2016) s'attache à corriger cette limite, grâce à une approche expérimentale utilisant une simulation à événements discrets, pour identifier précisément la valeur des facteurs à utiliser dans le calcul des zones du buffer. Dans un cas comme dans l'autre, le dimensionnement des buffers est une opération qui ne peut pas être réalisée manuellement. La question du couplage à un outil de calcul apparait alors comme nécessaire pour une exploitation opérationnelle en entreprise.

L'étape 3 de la méthode consiste, quant à elle, à réaliser l'ajustement des niveaux de buffer en fonction de l'évolution des attributs de l'article. En effet, en environnement VUCA, le dimensionnement du buffer devient obsolète rapidement. Il doit donc être ajusté dynamiquement pour pouvoir s'adapter. Chaque zone des buffers de stocks peut ainsi être recalculée soit, par une modification directe de ses paramètres de dimensionnement (le profil de buffer, la CMJ, le DLT, la MOQ, et le cycle de commande) si l'évolution a vocation à durer, soit par un réajustement périodique (ou facteur d'ajustement planifié) pour s'adapter à des variations de court - moyen terme. Dans ce dernier cas, il s'agit de traiter des effets dus à la saisonnalité, à des lancements et arrêts de produits ou à des campagnes de promotions qui vont être pris en compte dans le

7. Donnée d'entrée du DDOM envoyée par le DDS\&OP. 
redimensionnement du buffer par l'utilisation d'un facteur d'ajustement de la demande (Demand Adjustment Factor - DAF). Ce coefficient, qui est appliqué à la CMJ, est déterminé empiriquement par l'entreprise. Il n'existe, à notre connaissance, aucune technique formalisée par les auteurs pour le déterminer, mais l'utilisation d'une technique de gestion dynamique de stocks pourrait être envisagée. L'observation en temps réel des consommations permettrait par exemple de détecter une variation importante de celles-ci et donc la possibilité de réagir en amont, comme l'ont montré Pujo Doumani et Khader. (2016). Cela impliquerait pour le DDMRP la mise en place d'un outil informatique permettant de définir la valeur du coefficient en fonction de ces observations en temps réel.

Les trois étapes présentées ci-dessus sont cruciales pour une bonne utilisation de la méthode. Chaque positionnement et chaque dimensionnement de buffer auront un impact direct sur les décisions prises par les gestionnaires de production. Si cette méthodologie, décomposée en trois étapes distinctes, guide l'implémentation de la méthode, elle nécessite néanmoins un jeu d'essai-erreur et de tâtonnement, approche qui ne nous semble pas robuste pour un déploiement global rapide.

\section{Aspect opérationnel du DDMRP - les étapes 4 et 5 de la méthode}

L'attrait d'une telle méthode, pour les gestionnaires de production, s'appuie sur sa capacité à détecter et à prioriser les ordres d'achat et de production à lancer, tout en permettant une visualisation en temps réel de la situation de ces ordres. Ainsi la méthode combine à la fois la fonction de planification et d'exécution (les étapes 4 et 5 de la méthode) permettant un pilotage opérationnel au jour le jour.

La planification des ordres (étape 4) est réalisée chaque jour, pour chaque buffer, grâce à une équation nommée « équation de flux disponible », qui permet de déterminer si un ordre doit être lancé. Elle prend en compte la quantité en stock, la quantité en cours 
de commande et la demande qualifiée (ventes pour les produits finis ou besoins pour les produits intermédiaires) (Ptak et Smith, 2016, 149-150).

Flux disponible $=$ Stock physique + Approvisionnement - Demande qualifiée

L'équation intègre des temporalités différentes. Le stock physique correspond à la quantité en stock à la date du flux disponible $(t)$. L'approvisionnement - ou la quantité en cours de réapprovisionnement - est identifié sur une période (T : DLT) correspondant au délai découplé, c'est-à-dire au délai nécessaire pour acquérir l'article une fois un ordre d'achat ou de production passé. La demande qualifiée est définie comme la somme de la demande du jour, des demandes passées non satisfaites et à honorer et des pics de demande. Un pic de demande est, quant à lui, défini comme une demande qui dépasse un certain seuil (fixé par l'entreprise) sur un horizon spécifique (lui aussi fixé par l'entreprise). Des recommandations existent pour faciliter la fixation de ce seuil ( $50 \%$ de la zone rouge) et cet horizon (1 jour + délai découplé), mais il n'y a à notre connaissance aucune approche permettant un calcul précis de ces paramètres. La demande qualifiée est alors déterminée sur une période $\left(\mathrm{T}^{\prime}:\left[t-r_{i} ; t+h_{i}\right]\right):$ la date de début $\left(t-r_{i}\right)$ correspond à la date $t$ moins le nombre de jour de retard des commandes passées non honorées $\left(r_{i}\right)$; la date de fin $(t+h)$ correspond à la date $t$ plus le nombre jour composant l'horizon de pic de commande $\left(h_{i}\right)$.

Si le résultat de l'équation de flux disponible est inférieur ou égal au « Top du Jaune » (la somme des zones jaune et rouge du buffer), alors il est recommandé de créer un ordre de fabrication $(\mathrm{OF})$ ou d'achat (OA) égal à la différence entre le « Top du Vert» (la somme des zones verte, jaune et rouge), qui représente la taille du buffer, et le résultat de l'équation (cf. exemple 2). Ce calcul est effectué à tous les niveaux de la nomenclature de l'article bufférisé jusqu'à un autre article lui aussi bufférisé. La synchronisation entre 
les composants qui faisait la force du MRP est ai dépendance entre les articles par la mise en place de buffers afin de réduire la variabilité. Le gestionnaire de production va alors pouvoir choisir les ordres à lancer en fonction du pourcentage de remplissage de chaque buffer, celui-ci correspondant au rapport entre le flux disponible et le « Top du Vert». Grâce à ce pourcentage, il a en effet à sa disposition une liste d'ordres (approvisionnement ou production) classés par ordre de criticité, lui simplifiant la prise de décision, notamment en situation de capacité limitée.

Exemple 2. Itération du calcul du flux disponible.

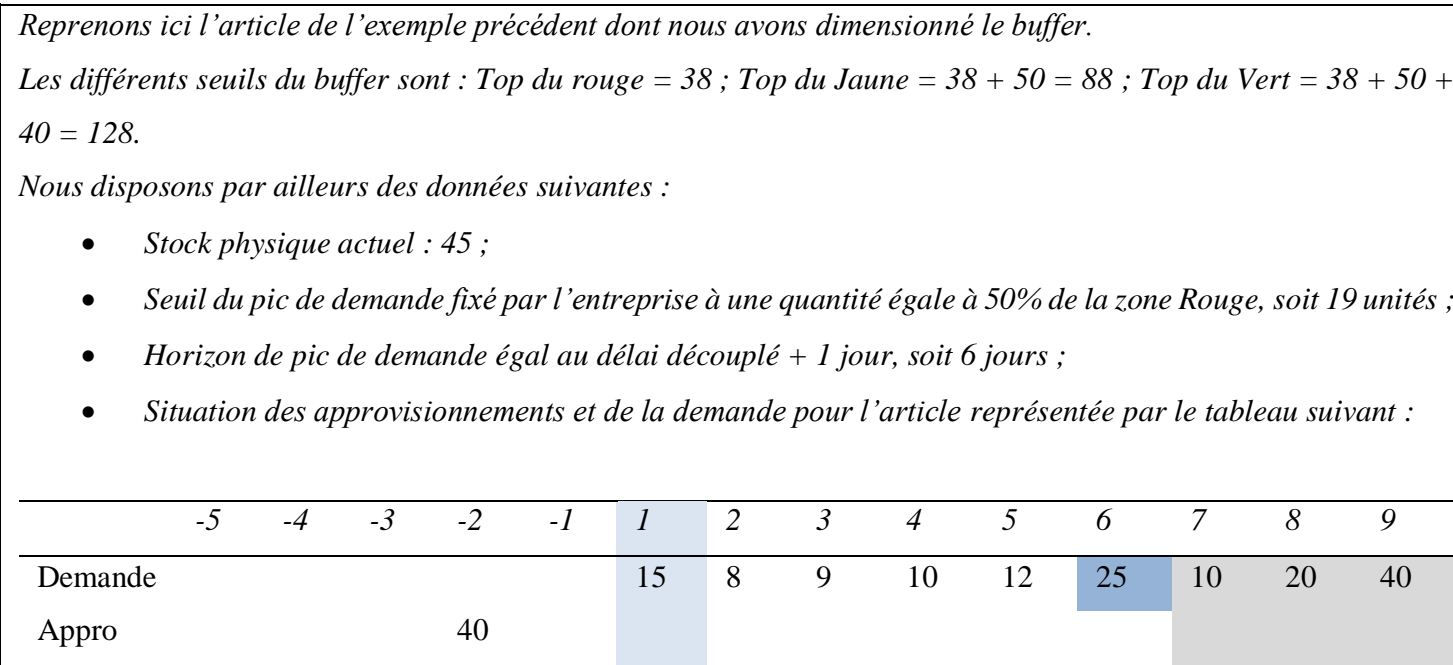

Jour 1 :

Nous avons :

- $\quad$ Stock $=45$

- Approvisionnement sur le DLT $=40$

- Demande qualifiée $=15+25$, avec 15 qui correspond à la demande du jour et 25 qui constitue le pic de demande sur l'horizon de 6 jours

Le flux disponible est donc égal à : $45+40-(15+25)=45$. Ce flux disponible étant inférieur au Top du Jaune (88), il faut donc créer un ordre de production de : 128 (Top du Vert) $-45=83$ unités, qui est planifié pour être livré dans 5 jours (pour le 6).

Le calcul du pourcentage de remplissage du buffer est de $: 45 / 128$ (Top du Vert) $=35 \%$ (Jaune)

Le niveau de stock de l'article, à la fin du jour 1, est quant à lui égal à : 45-15=30 unités.

Jour 2 :

\begin{tabular}{lllllllllllllll}
\hline & -5 & -4 & -3 & -2 & -1 & 2 & 3 & 4 & 5 & 6 & 7 & 8 & 9 & 10 \\
\hline $\begin{array}{l}\text { Demande } \\
\text { Appro }\end{array}$ & 83 & & & & & 8 & 9 & 10 & 12 & 25 & 10 & 20 & 40 & 10 \\
\end{tabular}


Pour le deuxième jour, l'horizon de calcul s'est déplacé d'une journée : le 7 du mois fait désormais parti de l'horizon. L'ordre de production que nous avons créé le jour précèdent apparait comme une livraison à recevoir. Nous constatons également que nous devons recevoir pour une quantité de 40 unités à la fin du jour.

Le flux disponible est égal à : $30+(83+40)-(8+25)=120$. Le flux disponible est supérieur au Top du Jaune, il n'y a rien à faire. Nous pouvons effectuer le calcul du pourcentage de remplissage du buffer : 120/128 = 93,75\% (Vert).

Le niveau de stock de l'article, à la fin du jour 2, est quant à lui égal à : $30+40-8=62$ unités.

L'étape 4 de planification des ordres s'appuie sur la gestion du flux disponible et du niveau de criticité qui en découle, mais ne prend pas en compte la situation actuelle du stock des différents articles. La phase «exécution » (étape 5), permet un suivi des ordres lancés par une priorisation visuelle grâce à un code couleur et par un système d'alertes. Deux types d'alertes sont utilisés (Ptak et Smith, 2011, 2016). Les premières, dites alertes de statut de buffer, sont composées des alertes sur stock physique actuel, conçues pour prévenir les gestionnaires de production des problèmes de disponibilité du stock des articles stratégiques, et des alertes projetées visant à anticiper cette situation. Les secondes, appelées alertes de synchronisation, mettent en avant la non-disponibilité de l'ensemble des éléments nécessaires à la réalisation d'un article à un temps $t$.

Le déclenchement d'une alerte sur stock physique actuel s'appuie sur la quantité physique en stock. Le code couleur pour le suivi des ordres reste le même que celui utilisé pour la planification mais sa signification change. Ainsi, le niveau de stock est considéré être dans la zone verte lorsqu'il est supérieur à la zone rouge de planification. La zone jaune et rouge d'exécution représentent alors chacune $50 \%$ de la zone rouge de planification, la partie supérieure correspondant à la zone jaune. Une alerte est déclenchée lorsque le stock passe dans la zone rouge du point de vue exécution. Il faut alors garder à l'esprit la distinction entre la fonction de génération des ordres et celle de suivi : être dans le vert du côté de la planification ne veut pas forcément dire que c'est le cas du côté de l'exécution. La priorisation des alertes s'effectuera dans ce cas en fonction du niveau de 
stock par rapport au « Top du Jaune » d'exécution. L'ordonnanceur (ou l'approvisionneur) dispose alors d'un suivi d'ordres classés par priorités pour déterminer ceux qui ont besoin d'être traités en urgence. Le DDMRP propose ainsi un pilotage par l'exception grâce à un management visuel qui permet une prise de décision plus simple et plus rapide promettant alors une amélioration des résultats de la chaîne logistique de l'entreprise.

En conservant l'historique de la position du stock, il est possible d'évaluer la performance du buffer au cours du temps et ainsi identifier s'il est bien dimensionné. Le buffer est considéré comme adapté s'il ne s'écarte que rarement de ce que l'on appelle l'intervalle optimal de stock dans lequel se trouve le niveau de stock objectif ${ }^{8}$. Cet intervalle est égal à l'écart entre la valeur correspondante au « Top du Rouge » et celle correspondante au «Top du Rouge » auquel s'ajoute la zone verte.

En synthèse, le DDMRP apparaît comme une méthode visant à fournir un pilotage au jour le jour qui simplifie la prise de décisions des gestionnaires de production. Les choix effectués sont de court terme et se positionnent à mi-chemin entre le calcul des besoins nets et l'ordonnancement des ordres générés par ce calcul. Il s'agit de répondre aux mêmes questions que les méthodes de gestion de production classiques à savoir : quoi, combien et quand produire ou approvisionner, tout en ajoutant une dimension de priorisation des différentes tâches par un management par l'exception grâce aux apports visuels de la méthode. Pour une utilisation optimale et fiable de celle-ci, la qualité du « paramétrage » préalable apparait comme essentielle puisque les opérateurs suivent uniquement des indications de couleurs sans avoir de vision globale de la situation. Or le paramétrage de certaines variables de calcul, comme les facteurs de délai ou de variabilité

8. Le niveau de stock objectif est égal à : Zone Rouge + Zone Verte / 2. 
mais aussi les seuils et horizons de pic demande ou encore les facteurs d'ajustement de la demande, repose, nous l'avons vu, sur une démarche très empirique dépendant d'un jugement qui, s'il peut s'avérer de bon sens, reste arbitraire. Des outils informatiques dédiées à la méthode semblent alors nécessaires pour, d'une part, garantir la prise en compte de cet environnement VUCA (dimensionnement et ajustement des buffers) et, d'autre part, déployer la méthode à un niveau opérationnel (couleur des zones et calcul périodique de l'équation de flux disponible). Par ailleurs, la méthode semble connaître une évolution progressive dans sa construction théorique : alors que seule la partie court terme était traitée dans l'ouvrage originel de Ptak et Smith (2011), de nouvelles fonctionnalités s'ajoutent progressivement au système Demand Driven (Smith, Ptak et Ling, 2017 ; Martin et al, 2018). Elles permettent la constitution d'un système complet de pilotage intégrant également les aspects de moyen - long terme, notamment les problématiques capacitaires et de calcul de la demande. Cependant les relations entre les différents niveaux décisionnels sont pour le moment peu stabilisées et documentées ; pourtant la gestion de ces problématiques est centrale pour le pilotage de la production de l'entreprise.

\section{L'attractivité du DDMRP : une étude exploratoire portant sur 30 cas}

La méthode DDMRP semble novatrice sur de nombreux aspects, mais s'avère encore à ce jour (i) peu stabilisée, la théorie se construisant progressivement, (ii) peu formalisée, certains éléments de calculs apparaissant très empiriques et (iii) peu documentée en raison du nombre réduit de travaux scientifiques sur le sujet. Les conditions de sa mise en œuvre, son périmètre d'utilisation, les typologies d'entreprises concernées, comme les réelles performances pratiques de la méthode, sont peu connues. Ce faible retour d'expériences peut être un frein au développement mais, actuellement, nous constatons néanmoins un attrait pour cette méthode, relayé par des éditeurs développant une offre adaptée (évoquée 
dans la suite de l'article). Qu'est-ce qui peut donc pousser actuellement certaines entreprises à adopter cette méthode ?

Après une présentation synthétique des premiers travaux académiques traitant de la méthode $(i)$, nous présenterons notre démarche méthodologique (ii).

\section{Revue de littérature}

A partir d'une consultation des bases de données EBSCO, Springer Link, ScienceDirect, Sage, Hal, ainsi que Google Scholar, nous avons ainsi identifié comme pertinents 3 articles scientifiques, 2 thèses et 8 communications traitant de DDMRP depuis 2015 . Trois axes de recherche semblent se dessiner autour du paramétrage, de la performance et des mécanismes de planification générés par l'adoption de la méthode (tableau 2).

Tableau 2. Cartographie des articles, communications et thèses traitant du DDMRP selon la méthodologie utilisée et la problématique soulevée.

\begin{tabular}{|c|c|c|c|c|}
\hline \multirow{2}{*}{\multicolumn{2}{|c|}{ Méthodologie }} & Qualitative & Quantitative & Expérimentale \\
\hline & & Etude de cas unique & Modélisation & Simulation / Optimisation \\
\hline \multirow{3}{*}{ لِّ } & $\begin{array}{c}\text { Paramétrage : } \\
\text { positionnement et } \\
\text { dimensionnement des } \\
\text { buffers }\end{array}$ & & & $\begin{array}{c}\text { Miclo (2016) } \\
\text { Jiang et Rim (2017) } \\
\text { Bayard et Grimaud (2018) }\end{array}$ \\
\hline & $\begin{array}{c}\text { Performance : comparaison } \\
\text { avec MRP et/ou Kanban ; ou } \\
\text { situation antérieure. }\end{array}$ & $\begin{array}{c}\text { Ihme (2015) } \\
\text { Ihme et Stratton (2015) } \\
\text { Kortabarria et al (2018) }\end{array}$ & & $\begin{array}{c}\text { Ihme (2015) } \\
\text { Ihme et Stratton (2015) } \\
\text { Miclo et al (2015) } \\
\text { Miclo (2016) } \\
\text { Miclo et al (2016) } \\
\text { Bayard et Grimaud (2018) } \\
\text { Miclo et al (2018) } \\
\text { Shofa } \text { et al (2018) }\end{array}$ \\
\hline & $\begin{array}{l}\text { Mécanisme de } \\
\text { planification }\end{array}$ & & Baptiste et Pellerin (2018) & \\
\hline
\end{tabular}

La plupart de ces travaux s'intéresse à l'aspect technique du DDMRP en utilisant notamment la simulation et/ou la modélisation comme méthodologie de recherche. Miclo et al (2018), par exemple, ont montré que la méthode permettait une amélioration du taux de service par rapport au Kanban et au MRP. Quelques travaux s'attachent, par une approche exploratoire, à étudier la manière dont la méthode peut être implantée (Ihme, 
2015) et à monter l'impact positif du DDMRP sur la performance opérationnelle d'une organisation (Kortabarria et al, 2018). Cependant aucune des recherches n'aborde la question des raisons poussant les entreprises à choisir cette voie.

\section{Design de recherche, modalité de recueil des données et caractéristiques de}

\section{l'échantillon retenu}

Pour traiter cette question, nous nous inscrivons dans le paradigme épistémologique interprétativiste. L'objectif est bien de comprendre quelles sont les motivations des entreprises pour la mise mettre en œuvre du DDMRP à partir de notre perception et donc d'en déduire des propositions de recherche (Giordano, 2003). Notre travail se base alors sur une approche qualitative à visée exploratoire qui s'appuie sur les informations d'un échantillon de 30 entreprises ayant déjà implanté ou étant en cours d'implantation de la méthode.

La collecte de données a été réalisée à partir de deux sources d'informations. La première est constituée de documents présentant les retours d'expériences d'entreprises utilisant la méthode. Ces documents sont des supports utilisés lors de communications à des conférences spécialisées réunissant des professionnels de la logistique. L'accès à ces cas n'est limité par aucune clause restrictive de confidentialité ; ils sont disponibles en libre-accès sur le site internet du Demand Driven Institute. Il convient cependant de prendre du recul par rapport à l'ensemble des informations présentes dans ces documents, l'objectif de cet organisme étant de promouvoir la méthode en mettant en avant les réussites. Nous considérons tout de même cette source d'information comme pertinente puisque nous ne cherchons pas à l'utiliser pour évaluer l'intérêt du DDMRP. Sur les 34 cas à notre disposition (et 56 entreprises identifiées comme ayant initié un projet), nous avons choisi d'exploiter uniquement ceux pour lesquels des informations relatives aux motivations des entreprise étaient disponibles ; cela représente 26 cas. 
La deuxième source d'information trouve son origine dans des échanges avec des praticiens de quatre entreprises, que nous nommerons Entreprise 1, 2, 3 et 4. Ces échanges ont eu lieu lors d'un workshop d'une journée et ont permis de questionner les entreprises sur leurs motivations quant à l'adoption du DDMRP. Cet apport nous permet de compléter les informations issues des documents du Demand Driven Institute.

Nous obtenons ainsi un échantillon de 30 entreprises dont les caractéristiques sont décrites dans le tableau 3. Celui-ci recense le secteur d'activité des entreprises, leur localisation, leur taille, leur mode de réponse au marché, et les fonctions logistiques concernées par la méthode (approvisionnement, production, et/ou distribution). Ainsi, la méthode, née aux Etats-Unis où 12 cas sont référencés, touche également la France avec 12 cas, et dans une moindre mesure d'autres pays tels que le Royaume-Uni et l'Espagne, mais également la Colombie. Le DDMRP ne semble donc pas se cantonner à ses frontières américaines et à des pays anglophones, mais poursuit un déploiement à un niveau international.

Tableau 3. Cartographie des cas étudiés.

\begin{tabular}{|c|c|c|c|c|c|}
\hline Entreprise & Secteur & Pays & Taille & Environnement & Fonction \\
\hline Arkopharma & Industrie Pharmaceutique & FR & ETI & MTS & $\mathrm{A}-\mathrm{P}$ \\
\hline Allergan & Industrie Pharmaceutique & US & ETI & MTS & $\mathrm{A}-\mathrm{P}-\mathrm{D}$ \\
\hline Avigilon & Industrie Electrique et Electronique & US & ETI & MTS & $\mathrm{P}-\mathrm{D}$ \\
\hline Bernard Controls & Industrie Electrique et Electronique & FR & ETI & MTO/MTS & $P-D$ \\
\hline BT Group & Industrie Electrique et Electronique & GB & GE & MTS & $\mathrm{A}-\mathrm{P}$ \\
\hline Coasa & Métallurgie / Aerospace & $\mathrm{ES}$ & ETI & MTO & $\mathrm{A}-\mathrm{P}$ \\
\hline Crylor & Métallurgie & FR & PME & MTO/ETO & $\mathrm{A}-\mathrm{P}$ \\
\hline Entreprise 1 & Autres Industries & FR & ETI & MTS/MTO & $\mathrm{A}-\mathrm{P}$ \\
\hline Entreprise 2 & Industrie Electrique et Electronique & FR & PME & MTS/MTO & $\mathrm{P}-\mathrm{D}$ \\
\hline Entreprise 3 & Autres Industries & FR & ETI & MTO & $\mathrm{P}$ \\
\hline Entreprise 4 & Industrie Electrique et Electronique & FR & ETI & MTS & $\mathrm{A}-\mathrm{P}$ \\
\hline Figeac Aero & Métallurgie / Aerospace & FR & ETI & ATO & $\mathrm{A}-\mathrm{P}-\mathrm{D}$ \\
\hline Forge USA & Métallurgie & US & PME & MTO & $\mathrm{P}-\mathrm{D}$ \\
\hline Kormotech & Agroalimentaire & UA & ETI & MTS & $\mathrm{A}-\mathrm{P}-\mathrm{D}$ \\
\hline LGI & Travaux d'impression & US & PME & MTO/MTS & $\mathrm{P}-\mathrm{D}$ \\
\hline Lightspeed Technologies & Industrie Electrique et Electronique & US & - & MTS & $\mathrm{A}-\mathrm{P}$ \\
\hline Miba Bearing & Métallurgie & US & ETI & MTO & $\mathrm{P}$ \\
\hline MIC & Textile/ Habillement & $\mathrm{CO}$ & GE & MTS/Retail & $\mathrm{A}-\mathrm{P}-\mathrm{D}$ \\
\hline Michelin & Automobile & FR & GE & MTS & $\mathrm{A}-\mathrm{P}$ \\
\hline Oregon Freeze Dry & Chimie/Parachimie & US & ETI & MTS & $\mathrm{A}-\mathrm{P}-\mathrm{D}$ \\
\hline Perma-Pipe & Métallurgie & US & ETI & MTO & D \\
\hline
\end{tabular}




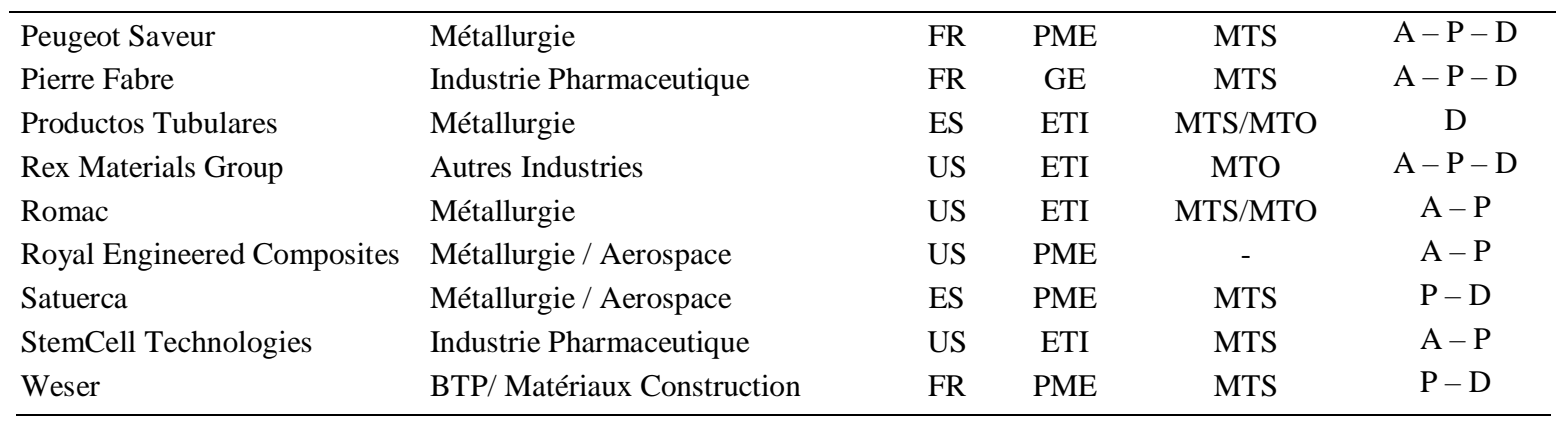

Pays : FR : France ; CO : Colombie ; ES : Espagne ; GB : Royaume-Uni ; UA : Ukraine ; US : Etats-Unis.

Taille : PME : Petite et Moyenne Entreprise ; ETI : Entreprise Taille Intermédiaire ; GE : Grande Entreprise.

Environnement: MTO: Make to Order; MTS: Make to Stock; ETO: Engineering to Order.

Fonction : A : Approvisionnement ; P : Production ; D : Distribution.

De plus, la méthode semble séduire aussi bien de petites entreprises que de grosses structures, dans des secteurs d'activités très variés et avec des modes de réponse au marché différents (sur stock, à la commande ou un mix des deux pratiques). Ainsi, nous trouvons aussi bien une grande entreprise de l'industrie pharmaceutique produisant sur stock (Pierre Fabre) qu'une PME de la métallurgie produisant à la commande (Forge USA). Quant au périmètre de déploiement interne de la méthode, il s'avère aussi très hétérogène : si la majorité des entreprises l'utilise pour gérer la production (28 cas sur 30), la méthode peut aussi être mobilisée sur la partie approvisionnement ou distribution, voir les trois activités. Ainsi pour 26 cas, il y a au moins deux fonctions déployées sous DDMRP et, pour 8 d'entre eux, les trois fonctions. La méthode s'inscrit alors dans une logique d'organisation d'entreprise reposant sur la notion de logistique intégrée?

La présentation de notre échantillon ne permet pas d'identifier un environnement précis d'implémentation de la méthode, le DDMRP ne semblant pas séduire un type particulier d'entreprises. Il est d'ailleurs remarquable de constater que les développeurs de la méthode ne proposent pas dans leurs ouvrages (Ptak et Smith, 2011 ; Ptak et Smith,

9. Assurer la synchronisation des processus des fonctions d'Achat, de Production et de Distribution dans le but d'éviter les ruptures et les sur-stocks (Colin, 2015). 
2016), une description des entreprises spécifiquement concernées par le DDMRP. Est-ce à dire que la méthode est universelle et peut fonctionner efficacement dans tous les environnements ?

\section{L’attractivité du DDMRP : analyse et résultats}

L'étude de nos 30 cas montre une raison initiale à la mise en œuvre du DDMRP : le souhait pour les entreprises de remplacer une méthode de gestion défaillante, ne permettant pas d'atteindre les objectifs escomptés en termes de taux de service et de niveau de stocks. Toutes les méthodes «classiques »sont concernées : le MRP chez Allergan, Lightspeed Technologies, Michelin, Entreprise 1, StemCell Technologies ... ; le Kanban chez Bernard Control ; ou encore la Théorie des Contraintes chez Avigilon, Miba Bearing ... Une autre approche de pilotage est souhaitée, en particulier par les personnes au contact quotidien des problèmes opérationnels qui sont à l'initiative du changement : responsable industriel, planificateur ou ordonnanceur. C'est le cas notamment d'entreprises comme Entreprise 1, Entreprise 2, StemCell Technologies, Allergan.

L'analyse des cas nous permet par ailleurs de formuler les propositions de recherche suivantes. Le DDMRP se serait imposé naturellement pour les entreprises de notre échantillon utilisant préalablement la théorie des contraintes $(P l)$. Les autres entreprises de notre panel ont été convaincues par les résultats obtenus par les premières $(P 2)$, ou par l'aspect «simpliste» de la méthode combiné au déploiement d'outils informatiques adaptés (P3).

\section{P1 : Un prolongement de la théorie des contraintes (TOC) pour les praticiens}

Les premières entreprises ayant implanté le DDMRP sont localisées aux Etats-Unis. Afin de comprendre ce qui les a motivées à être des pionnières sur ce sujet, nous nous sommes 
concentrés sur les 12 cas américains de notre échantillon. Nous avons ainsi identifié 8 entreprises qui, avant de migrer vers le DDMRP entre 2010 et 2013, utilisaient la théorie des contraintes et sa logique de synchronisation Drum - Buffer - Rope (Goldratt et Fox, 1986 ; Schragenheim et Ronen, 1990 ; Watson, Blackstone et Gardiner, 2007), appelé couramment DBR. La méthode DDMRP peut leur être apparue comme une suite logique : la théorie des contraintes proposant, en effet, de positionner stratégiquement des stocks de découplage, appelés également buffer, au sein des organisations pour limiter la conséquence des variabilités inhérentes à tout système (Goldratt et Cox 1993, Ye et Han, 2008). Ceux-ci doivent être positionnés soit devant les goulets (ou goulots) d'étranglement (ou contraintes) qui sont des ressources dont la capacité est inférieure ou égale à la demande du marché, et qu'il convient de protéger des aléas en amont; soit à la fin du processus pour assurer les délais de livraison des commandes en les protégeant contre les aléas de production sur le goulet et en aval de celui-ci ${ }^{10}$.

De plus, il apparaît que 3 des entreprises américaines (Avigilon, Perma Pipe et Miba Bearing), mais aussi 2 espagnoles (Coasa et Productos Tubulares) ont eu recours à la même société de conseil (Constraints Management Group, CMG) pour les aider dans la mise en place du DDMRP. Cette société, fondée en 1997 par Debra Smith et Chad Smith, est historiquement spécialisée dans l'implémentation et la mise en œuvre de la théorie des contraintes pour les entreprises industrielles ${ }^{11}$. Nous émettons alors l'hypothèse d'un lien étroit entre la théorie des contraintes et le DDMRP, qui serait vécu par les praticiens comme un prolongement de celle-ci.

10. Fawcett et Pearson (1991) ou Spencer (1991) préconisent l'utilisation d'un troisième type de stocks tampons (buffer) pour protéger les opérations d'assemblage dans les parties noncontraintes de la production. Cependant, il n’y a pas unanimité à ce sujet.

11. http://thoughtwarepeople.com/about-us/ 
En France, toutefois, la philosophie de la théorie des contraintes n'a pas connu un succès probant. Peu d'entreprises ont mis en œuvre cette approche. Il lui a été reproché, d'un point de vue pratique, de proposer peu d'outils pour mettre en œuvre la méthode DBR et, d'un point de vue théorique, de ne pas apporter de réponses claires et satisfaisantes à certaines difficultés. Il semble donc étonnant de constater que la méthode DDMRP séduise autant en France, alors que la méthode DBR y a été un échec. Quelles sont les raisons supplémentaires qui pourraient expliquer cet engouement?

\section{P2 : Des résultats prometteurs attirant les entreprises européennes}

Les premiers résultats positifs de la méthode sont apportés par les entreprises pionnières (celles évoquées au point précédent) à partir de l'année 2013. Ces résultats partiels semblent être en accord avec les promesses émises par le DDMRP : une amélioration du taux de service, une diminution globale du niveau des stocks et un pilotage simplifié de la production (tableau 4).

Tableau 4. Résultats des entreprises pionnières du DDMRP.

\begin{tabular}{lccc}
\hline \multicolumn{1}{c}{ Entreprise } & Taux de service & Niveau de stock & Autres \\
\hline Avigilon (2013) & $99 \%$ & NC & \\
Forge USA (2013) & $+87 \%$ & NC & Meilleure visibilité sur le processus de fabrication. \\
LG International (2011) & $+14 \%$, atteint $97 \%$ & $-50 \%$ & \\
Oregon Freeze Dry (2013) & $100 \%$ ou 99,6\% & $-30 \%$ à $-60 \%$ & Expéditions fiabilisées, attente camion 3-5 jours au \\
Perma-Pipe (2013) & NC & NC & \\
Rex Materials Group (2011) & $98 \%$ & $-60 \%$ & Augmentation des ventes de 13\% en moyenne. \\
Romac (2012) & NC & NC & \\
Royal Engineered Composites & $98 \%$ & NC & \\
\hline
\end{tabular}

C'est à partir de ces résultats que les créateurs de la méthode vont communiquer afin de convaincre d'autres entreprises d'adopter le DDMRP. Ils s'appuient également 
désormais sur les résultats d'une thèse (Miclo, 2016), montrant que le DDMRP permet de mieux absorber la variabilité de la demande que les méthodes MRP et Kanban en garantissant un taux de service acceptable pour l'entreprise. Ces premiers résultats convaincants semblent être une des raisons poussant les entreprises françaises et européennes à choisir le DDMRP (Entreprise 1, 2, 3 et 4, Allergan, BT Group) ; elles espèrent obtenir les mêmes résultats en adéquation avec leurs objectifs.

\section{P3 : Des outils numériques au service d'un management simple et visuel}

En complément des résultats affichés par la méthode, la simplicité de celle-ci attire également. Le code couleur proposé par la méthode permet à l'ensemble des personnes impliquées dans le processus de production de parler le même langage : Vert ( « Je fais autre chose »), Jaune («Attention »), Rouge («Urgence »). Cette simplicité des termes permet une meilleure compréhension pour les gestionnaires de production et le pilotage visuel de la production attire des entreprises (Entreprise 1, Entreprise 4, StemCell Technologies) souhaitant gagner du temps lors de l'exécution des tâches quotidiennes de lancement d'OF et d'OA.

La méthode peut aussi rapidement devenir complexe à mettre en place à l'échelle d'une entreprise, en raison, d'une part, du nombre d'informations à traiter et, d'autre part, de l'aspect visuel à modéliser. C'est pourquoi il nous semble impossible de dissocier l'outil informatique de la méthode pour comprendre l'engouement autour du DDMRP. Nous constatons que sur l'ensemble de notre échantillon, 25 cas mentionnent l'outil qui a été mis en place au même moment que le DDMRP. Ce qui avait fait défaut à la méthode DBR ne semble pas être le cas pour le DDMRP puisque les créateurs proposent actuellement une liste de logiciels «compliant», c'est-à-dire validés par le Demand Driven Institute, en complément de la méthode. Deux types d'outils numériques sont proposés : des add-on à interfacer avec un outil type ERP (Entreprise Ressource 
Planning) ou une fonctionnalité supplémentaire intégrée directement à l'ERP. Ce couplage de la méthode à un outil informatique permet de combler aussi bien les attentes en termes d'opérationnalisation, mais également celles concernant la centralisation et la fiabilisation du pilotage de la chaîne logistique (Weser, Entreprise 4 et 3).

En synthèse, le tableau 5 propose une première grille de lecture empirique des motifs d'engouements autour de cette méthode. Basée sur un ensemble d'entreprises ayant mis en place la philosophie de la théorie des contraintes, la méthode a bénéficié d'accès à des terrains pour pouvoir tester une mise en production et connaître de premiers résultats. Le rayonnement du DDMRP a ainsi pu s'étendre, soutenu également par les éditeurs de solution informatique, des associations de supply chain et des cabinets de conseil.

Tableau 5. Synthèse des motivations des cas étudiés pour le choix du DDMRP.

\begin{tabular}{|c|c|c|c|}
\hline Entreprise & Hérités de la TOC (1) & Résultats (2) & Outils (3) \\
\hline Arkopharma & & $\mathrm{X}$ & $\mathrm{X}$ \\
\hline Allergan & & $\mathrm{X}$ & $\mathrm{X}$ \\
\hline Avigilon & $\mathrm{X}$ & & $\mathrm{X}$ \\
\hline Bernard Controls & & $\mathrm{X}$ & $\mathrm{X}$ \\
\hline BT Group & & $\mathrm{X}$ & $\mathrm{X}$ \\
\hline Coasa & $\mathrm{X}$ & & \\
\hline Crylor & & $X$ & \\
\hline Entreprise 1 & & $\mathrm{X}$ & $X$ \\
\hline Entreprise 2 & & $X$ & $X$ \\
\hline Entreprise 3 & & $\mathrm{X}$ & $\mathrm{X}$ \\
\hline Entreprise 4 & & $\mathrm{X}$ & $\mathrm{X}$ \\
\hline Figeac Aero & & $X$ & $X$ \\
\hline Forge USA & $\mathrm{X}$ & & \\
\hline Kormotech & & $\mathrm{X}$ & $\mathrm{X}$ \\
\hline LG International & $\mathrm{X}$ & & $\mathrm{X}$ \\
\hline Lightspeed Technologies & & $X$ & $\mathrm{X}$ \\
\hline Miba Bearing & $\mathrm{X}$ & & $\mathrm{X}$ \\
\hline MIC & $\mathrm{X}$ & & $\mathrm{X}$ \\
\hline Michelin & & $X$ & $\mathrm{X}$ \\
\hline Oregon Freeze Dry & $\mathrm{X}$ & & $\mathrm{X}$ \\
\hline Perma-Pipe & $\mathrm{X}$ & & \\
\hline Peugeot Saveur & & $X$ & $\mathrm{X}$ \\
\hline Pierre Fabre & & $\mathrm{X}$ & $X$ \\
\hline Productos Tubulares & $\mathrm{X}$ & & $\mathrm{X}$ \\
\hline Rex Materials Group & $\mathrm{X}$ & & \\
\hline Romac & $\mathrm{X}$ & & $\mathrm{X}$ \\
\hline Royal Engineered Composites & $\mathrm{X}$ & & $\mathrm{X}$ \\
\hline
\end{tabular}




\begin{tabular}{lll}
\hline Satuerca & $\mathrm{X}$ & $\mathrm{X}$ \\
StemCell Technologies & $\mathrm{X}$ & $\mathrm{X}$ \\
Weser & $\mathrm{X}$ & $\mathrm{X}$ \\
\hline
\end{tabular}

\section{Conclusion et perspectives}

Le DDMRP connait depuis quelques mois un engouement fort dans la communauté professionnelle. Des entreprises de toutes tailles, de tous secteurs d'activités et ayant des modes différents de réponses au marché, s'y intéressent et cela même si des limites à son déploiement peuvent être recensées. Le DDMRP apparaît ainsi non stabilisé sur de nombreux points, laissant à l'initiative des entreprises la définition de plusieurs paramètres conditionnant ses performances comme les facteurs de délai et de variabilité, le seuil et l'horizon de pic de demande et le facteur d'ajustement de la demande.

L'étude de 30 cas d'entreprises nous a permis de mettre en évidence que les faiblesses relatives aux méthodes de gestion de production utilisées ont poussé celles-ci à vouloir faire évoluer leur pilotage afin d'atteindre les objectifs de taux de service et de niveau des stocks souhaités. Les entreprises de notre échantillon semblent s'être tournées vers le DDMRP pour trois raisons. Les premières entreprises l'ayant mise en place utilisaient la théorie des contraintes, un courant auquel une partie des créateurs du DDMRP ont activement participé. Les premiers résultats communiqués sont en cohérence avec les promesses de la méthode permettant de rassurer et attirer de nouvelles entreprises. L'aspect simple et visuel de la méthode couplé à une solution informatique pouvant être interfacée avec les outils déjà utilisés en entreprise semble être une raison supplémentaire motivant les organisations à choisir le DDMRP. Ces résultats mériteraient toutefois d'être vérifiés auprès d'un échantillon plus large d'entreprises et, pour gagner en objectivité, à partir de données non contrôlées par le Demand Driven Institute.

Au-delà de cette piste de recherche, plusieurs autres perspectives nous semblent intéressantes à explorer au regard de nos résultats. Il s'agirait tout d'abord de définir la 
typologie des entreprises concernées par le DDMRP. Un travail pourrait aussi être mené pour confirmer ou infirmer les performances réelles de la méthode grâce à des études longitudinales. La clarification du positionnement de la méthode dans un système de pilotage court - long terme pourrait être également utile pour faciliter sa mise en œuvre. En effet, une des limites de notre contribution est l'apport d'un seul niveau de lecture concernant la délimitation des éléments associés au DDMRP, et non un apport critique concernant celle-ci. Par exemple, le fait que plusieurs étapes (1 à 3) semblent être effectuées à un niveau de décision tactique (DDS\&OP) et non opérationnel ou encore par l'approche réductrice utilisées par les auteurs pour justifier le développement du DDMRP. Le MRP et le Kanban ne sont pas les seules méthodes de gestion de production existantes. Nous avons notamment cité la TOC dans cet article mais des méthodes comme le CONWIP (CONstant Work In Process), POLCA (Paired cell Overlappings Loops of Cards with Authorization) ou WLC (WorLoad Control) existent également et peuvent répondre aux limites du MRP et du Kanban (Stevenson, Hendry et Kingsman, 2005). Il existe également de nombreuses adaptations du Kanban. Ainsi, 32 identifiées par Lage Junior et Fiho (2010) ont été développées pour dupliquer la logique de production japonaise à des environnements plus complexes. L'utilisation de takt-time variables pour des lignes de production « $U$ » (Fouzia, Pujo et El Khabous, 2015) est également une piste dans la prise en compte de l'incertitude de l'environnement.

Enfin, il serait pertinent de déterminer des techniques permettant de calculer scientifiquement les paramètres jusqu'alors choisis empiriquement, une piste que nous avons ouverte en suggérant une méthode de gestion dynamique de stocks pour déterminer la valeur du facteur d'ajustement de la demande. Le DDMRP n'en est qu'à ses balbutiements : effet de mode ou réelle innovation ?... Les travaux tant théoriques qu'empiriques permettront surement de répondre à cette interrogation. 


\section{Références}

Baptiste, Pierre, et Robert Pellerin. 2018. "Planification à court terme dans un système de production complexe géré en DDMRP." Papier présenté à la 12ème Conférence internationale de Modélisation, Optimisation et SIMulation (MOSIM). Toulouse, France.

Bayard, Stéphanie, et Frédéric Grimaud. 2018. "Enjeux financiers de DDMRP : une approche simulatoire." Papier présenté à la 12ème Conférence internationale de Modélisation, Optimisation et SIMulation (MOSIM). Toulouse, France.

Bennett, Nathan, and G. James Lemoine. 2014. "What a difference a word makes : Understanding threats to performance in a VUCA world." Business Horizons, May. 311-317. doi: 10.1016/j.bushor.2014.01.001.

Colin, Jacques. 2015. "La logistique: histoire et perspectives." Logistique \& Management, 23(4) : 25-40. doi :10.1080/12507970.2015.11758620

Deming, W. Edwards. 1975. "On Some Statistical Aids Toward Economic Production." Interfaces $5,4: 1-15$.

Fawcett, Stanley, and John Pearson. 1991. "Understanding and Applying Constraint Management in Today's Manufacturing Environments." Production and Inventory Management Journal, 3 : 46 - 55.

Forrester, Jay W. 1961. Industrial dynamics. M.I.T. Press.

Giordano Yvonne. 2003. Conduire un projet de recherche. Une perspective qualitative. EMS.

Goldratt, Eliyahu M. and Jeff Cox. 1993. Le but: l'excellence en production. $2^{\text {nd }}$ ed. AFNOR Gestion.

Goldratt, Eliyahu M. and Robert Fox. 1986. The Race. North River Press. 
Ihme, Mathias. 2015. "Interpretating and applying Demand Driven MRP, a case study". Nottingham Trent University.

Ihme, Mathias, and Roy Stratton. 2015. "Evaluating demand driven MRP : a case based simulated study." Paper presented at the International Conference of the European Operations Management Association. Neuchatel, Switzerland.

Jiang, Jingjing, and Suk-Chul Rim. 2017. "Strategic WIP Inventory Positioning for Maketo-Order Production with Stochastic Processing Times." Mathematical Problems in Engineering 2017. doi.org/10.1155/2017/8635979.

Kortabarria, Alaitz, Unai Apaolaza, Aitor Lizarralde, and Itxaso Amorrortu. 2018. "Material Management without Forecasting: From MRP to Demand Driven MRP." Journal of Industrial Engineering and Management, 11 (4) : 632-650. doi.org/10.3926/jiem.2654.

Lage Junior, Muris, and Moacir Godinho Filho. 2010. "Variations of the kanban system: Literature review and classification." International Journal of Production Economics, 125 (1): 13-21. doi.org/10.1016/j.ijpe.2010.01.009.

Lee, Hau L., V. Padmanabhan, and Whang Seungjin. 2004. "Information Distortion in a Supply Chain : The Bullwhip Effect." Management Science, 50 : 1875-1886. doi :10.1287/mnsc.1040.0266

Mackelprang, Alan W., and Manoj K. Malhotra. 2015. "The impact of bullwhip on supply chains : Performance pathways, control mechanisms, and managerial levers." Journal of Operations Management, 36 : 15-32. doi: 10.1016/j.jom.2015.02.003

Martin, Guillaume, Pierre Baptiste, Jacques Lamothe, Romain Miclo, et Matthieu Lauras. 2018. "Vers une cartographie de processus explicite pour le modèle Demand Driven Adaptive Enterprise." Papier présenté à la 12ème Conférence internationale de Modélisation, Optimisation et SIMulation (MOSIM). Toulouse, France. 
Miclo, Romain. 2016. "Challenging the "Demand Driven MRP” Promises : a Discret Event Simulation Approach." Université de Toulouse.

Miclo, Romain, Franck Fontanili, Matthieu Lauras, Lamothe, Jacques Lamothe, and Bernard Milian. 2015. "MRP vs. demand-driven MRP: Towards an objective comparison. " Paper presented at the Industrial Engineering and Systems Management (IESM). International Conference Seville, Spain. doi.org/10.1109/IESM.2015.7380288

Miclo, Romain, Franck Fontanili, Matthieu Lauras, Jacques Lamothe, and Bernard Milian. 2016. "An empirical study of Demand-Driven MRP." Paper presented at the 6th International Conference on Information Systems, Logistics and Supply Chain (ILS). Bordeaux, France.

Miclo, Romain, Matthieu Lauras, Franck Fontanili, Jacques Lamothe, and Steven A. Melnyk. 2018. "Demand Driven MRP: assessment of a new approach to materials management. " International Journal of Production Research, 1-16. doi: 10.1080/00207543.2018.1464230

Ohno, Taiichi. 1989. L'esprit Toyota. Masson.

Ounnar, Fouzia, Patrick Pujo, and Ilham El Khabous. 2015. "Experimental assessment of the productivity improvement when using U-shaped production cells with variable takt time." International Journal of Lean Six Sigma, 6 (1) : 17-38. doi.org/10.1108/IJLSS-07-2013-0038.

Packowski Josef. 2013. LEAN Supply Chain Planning : The New Supply Chain Management Paradigm for Process Industries to Master Today's VUCA World. CRC Press.

Pujo, Patrick, Ikram Doumani, et Selma-Afakh Khader. 2016. "Maitrise des stocks en logistique par l'observation de la consommation". Papier présenté aux $11^{\text {ème }}$ 
Rencontres Internationales de la Recherche en Logistique et Supply Chain (RIRL). Lausanne, Switzerland.

Ptak, Carol, and Chad Smith. 2011. Orlicky's material requirements planning. $3^{\text {rd }}$ ed. McGraw-Hill.

Ptak, Carol, and Chad Smith. 2016. Demand driven material requirements planning (DDMRP). Industrial Press, Inc.

Rim, Suk-Chul, Jingjing Jiang, and Chan Ju Lee. 2014. "Strategic Inventory Positioning for MTO Manufacturing Using ASR Lead Time." In Logistics Operations, Supply Chain Management and Sustainability, 441-456. EcoProduction. Springer.

Schonberger, Richard J. 1983. "Applications of Single-Card and Dual-Card Kanban." Interfaces, 13 (4) : 56-67.

Schragenheim, Eli, and Boaz Ronen. 1990. "Drum-buffer-rope shop floor control." Production \& Inventory Management Journal, 31 (3) : 18-22.

Shofa, Mohamad J., Armand O. Moeis, and N Restiana. 2018. "Effective production planning for purchased part under long lead time and uncertain demand : MRP Vs demand-driven MRP". IOP Conference Series : Materials Science and Engineering, 337 (1) : 012055. doi :10.1088/1757-899X/337/1/012055

Smith, Chad, Carol Ptak, and Dick Ling. 2017. "The Demand Driven Adaptive Enterprise Model : The case for a new system of enterprise management." Demand Driven Institute. https://www.demanddriveninstitute.com/demand-driven-adaptiveenterprise-m

Smith, Debra, and Chad Smith. 2013. Demand driven performance : using smart metrics. McGraw-Hill Education.

Spencer, Michael. 1991. "Using the Goal in an MRP system." Production and Inventory Management Journal, $1: 22-27$. 
Stevenson, M., L. C. Hendry, and B. G. Kingsman. 2005. "A review of production planning and control : the applicability of key concepts to the make-to-order industry." International Journal of Production Research, 43 (5) : 869-98.

Wang, Xun, and Stephen M. Disney. 2016. "The bullwhip effect : Progress, trends and directions." European Journal of Operational Research, 250 (3) : 691-701. doi : 10.1016/j.ejor.2015.07.022.

Watson, Kevin J., John H. Blackstone, and Stanley C. Gardiner. 2007. "The evolution of a management philosophy : The theory of constraints." Journal of Operations Management, 25 (2) : 387-402. doi.org/10.1016/j.jom.2006.04.004.

Ye, T., and W. Han. 2008. "Determination of buffer sizes for drum-buffer-rope (DBR)controlled production systems." International Journal of Production Research, 46 (10) : 2827-44. doi.org/10.1080/00207540600922948. 